\title{
INOVAÇÃO TECNOLÓGICA: TÉCNICAS E FERRAMENTAS APLICADAS AO PROJETO DE EDIFICAÇÕES
}

\section{TECHNOLOGICAL INNOVATIONS: TECHNIQUES AND TOOLS APPLIED IN BUILDING DESIGN}

\author{
Fabíola Azuma \\ Mestranda do Programa de Pós-Graduação em Construção Civil - UFPR \\ Centro Politécnico, CEP 81.531-980, Cx. Postal 19.011, Curitiba, Paraná. \\ Telefone: (41) 3361-3110 \\ e-mail: fabiolaazuma@yahoo.com.br

\section{Caroline Barp Zanchet Machado} \\ Mestranda do Programa de Pós-Graduação em Construção Civil - UFPR \\ Centro Politécnico, CEP 81.531-980, Cx. Postal 19.011, Curitiba, Paraná. \\ Telefone: (41) 3361-3110 \\ e-mail: zanchet@ufpr.br
}

\section{Maria do Carmo Duarte Freitas}

Professor Adjunto do Departamento de Ciências e Gestão da Informação - UFPR

Av. Prefeito Lothário Meissner, 634. Jardim Botânico, Campus III. CEP 80210.170, Curitiba, Paraná.

Telefone: (41) 360-4470/Fax (41) 360-4420

e-mail: mcf@ufpr.br

\section{Sergio Scheer}

Professor Adjunto do Departamento de Construção Civil - UFPR Centro Politécnico, CEP 81.531-980, Cx. Postal 19.011, Curitiba, Paraná. Telefone: (41) 3361-3218 / Fax: (41) 3361-3436

e-mail: scheer@ufpr.br

\section{Aloísio Leoni Schmid}

Professor Adjunto do Departamento de Arquitetura e Urbanismo - UFPR Centro Politécnico, CEP 81.531-980, Cx. Postal 19.011, Curitiba, Paraná.

Telefone: (41) 361-3068 / Fax (41) 361-3084

e-mail: iso@ufpr.br 
Resumo: apresentam-se as inovações tecnológicas incorporadas pela Indústria da Construção Civil ao Projeto de Edificações, visando às novas perspectivas de mercado de modo a oferecer maior produtividade, flexibilidade e qualidade nas construções. Observa-se que mudou o perfil do profissional, as ferramentas, técnicas e processos de trabalho para quem atua em projetos. O objetivo deste artigo é propor uma listagem dessas inovações tecnológicas encontradas no setor de projetos de edificações. Parte-se de uma revisão bibliográfica que oferecerá ao profissional do setor de projeto uma ferramenta de avaliação de seus conhecimentos sobre o novo na área em que atua. Há uma tendência na idealização de um projeto mais holístico e integrado, abrangendo todas as fases do ciclo de vida da construção, desde concepção até a manutenção e demolição.

Palavras-chave: Inovações tecnológicas, projetos de edificações, tecnologia da informação.

\begin{abstract}
Construction Industry in the Building Design are presented in this article taking into account new market views in order to offer high level of productivity, flexibility and quality in the construction. Changes in professional's profiles, tools, techniques and working procedures on projects have observed. The purpose of this article is to provide a listing of these technological innovations found in building design sector. The listing, based on the literature review, will offer to the design professional a tool for their knowledge evaluation about the new in their field of work. There is a tendency in the idealization of a more holistic and integrated project, covering all phases of the construction life cycle, from design to maintenance and demolition.
\end{abstract}

Keywords: technological innovations, building design, information technology.

\title{
1. INTRODUÇÃ̃O
}

A incorporação de inovações tecnológicas, no setor de projetos de edificações provocou impactos na maneira de projetar, gerenciar e executar um empreendimento. Essas inovações são decorrentes das novas demandas do mercado, que buscam maior qualidade, flexibilidade, melhorar o atendimento ao cliente, reduzir custos e re-trabalhos e maior produtividade. De forma a atender a essas expectativas, surgiram novos métodos e paradigmas para quem atua no desenvolvimento de projetos.

O processo tradicional de desenvolvimento de projeto desvinculado do processo construtivo é coisa do passado. A empresa do futuro não poderá ser negligente com as possibilidades de racionalizações, dos estudos alternativos de construção, dos arranjos espaciais adequados, das melhorias nas instalações e facilidades de manutenção e operação, da seleção de materiais de construção adequados. Enfim, considerar os fatores que afetam a qualidade da construção e que impacta na satisfação do cliente final. 
O novo paradigma de processo tem o projeto como o centro de todos os profissionais envolvidos na construção, portanto integrando os processos construtivos e as análises que conferem maior qualidade a obra. Nesse contexto, entram discussões referentes ao desenvolvimento sustentável, no qual o projeto é visto de uma forma ampla, considerando não só os aspectos de construção, mas também do meio ambiente.

$\mathrm{O}$ advento das aplicações computacionais e da Internet trouxeram um novo impulso a esse paradigma. É possível fazer análises, simulações e visualizações mais precisas e de melhor qualidade. A tecnologia também impulsionou a colaboração entre profissionais geograficamente distantes, possibilitando o desenvolvimento integrado de projetos com equipes remotas. No entanto, a adoção de novos métodos e ferramentas acarretou mudanças na formação do profissional e existe a necessidade da reconversão profissional.

Esta entre outras é a motivação para este artigo, que tem como meta apresentar as inovações tecnológicas encontradas no setor de projetos de edificações nas últimas décadas. Para tanto, apresenta-se o resultado de um levantamento bibliográfico englobando conceitos como qualidade, flexibilidade, engenharia simultânea, ferramentas de tecnologia da informação e desenvolvimento sustentável. Como produto dessa revisão bibliográfica, faz-se uma proposta de check-list sobre inovações tecnológicas em projetos baseado na listagem sobre inovações tecnológicas em canteiros de obras (Pozzobon et al., 1999).

\section{INOVAÇÃO TECNOLÓGICA APLICADA AO PROJETO DE EDIFICAÇÕES}

A inovação tecnológica é um processo iniciado a partir de uma idéia técnica e que se concretiza na geração de um novo produto ou processo produtivo (JACOSKI, 2003). Esse processo de inovação está, portanto, relacionado com a criatividade.

Para o setor da construção civil, no processo de inovação tecnológica, o projeto é um elemento estratégico. Fabrício (2002) complementa que nesta etapa são feitas as opções pela utilização de novos materiais e, sobretudo o detalhamento e a especificação de como empregá-los, além das soluções das interfaces desses novos materiais e componentes com o sistema construtivo.

Por tradição inovar na Indústria da Construção Civil é fruto de imposição de outros setores industriais. Fato que provocou mudanças na área de projetos de edificações. Os projetistas são pressionados a incorporarem critérios de qualidade e a buscarem ser competitivos. Para tanto, 
tiveram que se adequar com inovações referentes à flexibilidade dos produtos na fase de projeto e da elaboração deste com uso intenso das novas tecnologias de informação.

A transição do projeto em papel para o digital com a inserção de programas computacionais facilitou o processo de correção em projetos e suscitou os estudos para desenvolvimento de projetos mais flexíveis. No Brasil, os primeiros estudos sobre o tema surgem com as pesquisas de Brandão (2002). Joedicke (1979) apud Brandão (2002) afirma que flexibilidade de uma edificação diz respeito à sua capacidade de modificação da função sem modificação das partes construídas abrangindo a capacidade de planejar arranjos espaciais diversos, versáteis e reversíveis. Para Brandão (2002), a flexibilidade espacial possui estreita relação com o sistema estrutural, pois incorpora o conceito de versatilidade de posicionamento das paredes divisórias. As soluções tecnológicas e fatores que possibilitam maior variação espacial citadas pelo autor são: painéis leves (drywall); diversificação dos materiais de construção e acabamento; uso de lajes nervuradas - sistema estrutural que não restringe a divisão dos espaços internos tanto pela liberdade de arranjo das paredes quanto pelo teto plano (Nappi, 1993 apud Brandão, 2002); e, no âmbito internacional, a conversão de edifícios de escritórios em edifícios residenciais na década de 90.

Nesse contexto faz-se a seguir um resgate das ferramentas tecnológicas inseridas nos últimos anos que auxiliam o processo projetual, mostrando a evolução dos sistemas Computer Aided Design - CAD e as ferramentas para colaboração móvel.

\subsection{Sistemas Computacionais aplicados ao Projeto de Edifícios}

Os projetos de edifícios eram feitos através de lápis e papel, maquetes e fotografias. Com o surgimento e evolução dos recursos computacionais, os projetos passaram a ser expressos como: imagens fotorrealísticas, animações tridimensionais, multimídia interativa e realidade virtual, representando uma nova atitude sobre pensar o espaço físico na concepção e representação do processo projetual (CARVALHO \& ALMEIDA, 2002).

\subsubsection{Sistemas CAD 2D e 3D}

Existem diversos sistemas CAD no mercado em duas ou três dimensões (2D e 3D), mas nem sempre estes são concorrentes diretos. Genericamente, os sistemas são melhor habilitados a realizar uma variedade de tarefas, cada um se ajustando com maior ênfase em uma determinada aplicação (SOUZA \& COELHO, 2003). Os autores citados classificam os sistemas CAD pelo porte. No primeiro segmento os sistema de pequeno porte (Low-end) sistemas que utilizam apenas representações geométricas em duas dimensões. No seguinte, os 
de médio porte (Middle-end) - a classe de software em maior ascensão no mercado atualmente, tendo como características importantes a paramétrica e associativa. Paramétrica de forma que a alteração no valor numérico de um determinado parâmetro permite a atualização automática em todos os valores atrelados a ele. E associativa posto que permitem a geração automática de desenhos 2D, incluindo vistas e cotas para dimensionamento, a partir do modelo 3D, de modo que alterações no modelo 3D refletem automaticamente no desenho 2D. Já os sistemas CAD de grande porte (High-end) abrangem todos os recursos dos sistemas anteriores, adicionando: capacidade de modelagem híbrida; visualização realista ou fotográfica; e integração de sistemas (fabricação, simulação, análise, planejamento, etc CAM, CAE, CAPP, etc).

Na Construção Civil, a tecnologia do CAD3D pode reduzir problemas desde o entendimento pelo cliente do projeto solicitado até os da busca de resolução de problemas construtivos antes de começar a obra. A modelagem tridimensional torna o processo de projeto ágil, minimiza o esforço de compreensão do projeto dos envolvidos no processo de desenvolvimento e reduz o índice de erros na execução (PINHO et. al., 2003). A tecnologia de CAD3D trouxe benefícios para a compatibilização e coordenação de projetos, permitindo a substituição do processo de compatibilização através da superposição e análise de desenhos bidimensionais, explicitando as interferências físicas entre elementos construtivos.

Os avanços na indústria da manufatura (projetos colaborativos, planejamento de processos inteligentes e Computer-Integrated Manufacturing - CIM) proporcionaram uma reflexão para formular um paradigma análogo para Computer-Integrated Construction (CIC). Embora existam similaridades filosóficas, existem diferenças em relação a processos tecnológicos, fatores humanos, custos, comunicação, entre outras (VEERAMANI et al., 1998).

\subsubsection{Sistemas CAD4D}

Tanyer \& Aouad (2005) afirma que as pesquisas em visualização 4D começaram em 1990. McKinney e Fischer (1997) apud Tanyer \& Aouad (2005) dizem que os sistemas CAD4D representam o relacionamento entre informações de planejamento e informações CAD por meio de um filme 4D que visualmente retrata a seqüência da construção da edificação. Este tipo de sistema é de fato uma ferramenta para mudanças no processo de planejamento, projeto e estratégias de gerenciamento de construção das companhias.

Porém, Tanyer \& Aouad (2005) cita restrições ao modelo 4D que impedem que essa ferramenta seja absorvida totalmente, tais como: o tempo consumido na criação dos modelos 
4D e na sua atualização para cada situação ou nível de detalhe; falta de informações sobre o conhecimento da construção, equipamentos, recursos e interferências; e falta de integração com outros dados do projeto. Entretanto, segundo Tanyer \& Aouad (2005), a próxima geração de ferramentas 4D está sendo implementada para superar essas dificuldades e pesquisadores estão buscando a integração dos modelos 4D a banco de dados compatíveis com IFC (Industry Fondation Classes).

IFC é proposta da IAI (International Alliance for Interoperability) e consiste em um modelo de dados aberto e não proprietário para Arquitetura, Engenharia e Construção (AEC). O objetivo é a padronização de classes utilizadas em sistemas orientados a objetos baseados em modelos, permitindo que diferentes aplicativos (como CAD, orçamentação, planejamento, cálculo estrutural, etc), compartilhem dados (NASCIMENTO, 2004).

Os sistemas CAD mais recentes buscam incorporar as IFC's adotando o conceito de BIM (Building Information Modelling) e são chamados Virtual Building, Parametric Modelling ou Model-Based Design. Esses produtos constroem modelos de edificações com objetos paramétricos, como paredes, colunas e janelas e são fundamentados na modelagem baseada em objeto (TSE et al., 2005). Exemplos são o AllPlan da Nemetschek, o ArchiCAD da Graphisoft e, mais recentemente, o Revit da Autodesk.

O BIM e as IFC's são resposta a falta de integração das etapas de projeto, visando atender a interoperabilidade de sistemas, na linha da idéia de CADnD e CIC.

\subsubsection{Sistemas CADnD}

As ferramentas CADnD, segundo Tanyer \& Aouad (2005), abrangem diferentes dimensões de projeto, tais como custo, condições de entorno e dados relacionados a manutenção. Fu et al. (2006) e Marshall-Ponting \& Aouad (2005) afirmam que este modelamento n-dimensional atende dimensões como projeto, construção e manutenção, tornando-as explícitas. Tem o objetivo de possibilitar decisões considerando prazo, custo, acessibilidade, sustentabilidade, acústica, conforto térmico e visual, construtibilidade e manutenção e assim constituindo uma ferramenta holística de tomada de decisão.

Os sistemas $\mathrm{nD}$ ou multidimensionais possibilitam análises dos impactos das características de projeto nos prazos, custos, manutenção e entre outros fatores. $\mathrm{O}$ valor da ferramenta $\mathrm{nD}$ consiste na habilidade em representar e manipular um grande volume, além de diferentes tipos de informação rapidamente. Dessa forma, pode destacar os conflitos em projetos e contribuir para o desenvolvimento de um pensamento transdisciplinar para projetistas e construtores. As 
oportunidades identificadas na implementação de ferramentas $\mathrm{nD}$ consistem: na captura, utilização e transferência do conhecimento, promoção da educação e treinamento, melhoria nos processos de tomada de decisão, colaboração entre projetistas, foco nos anseios dos clientes do projeto, modelagem computadorizada, otimização de padrões e interoperabilidade. Promove a comunicação entre os envolvidos, aumenta a compreensão dos diversos pontos de vista e auxilia no desenvolvimento de um projeto e construção mais holístico (MARSHALLPONTING \& AOUAD, 2005).

\subsection{Tecnologias para Trabalhos Colaborativos}

$\mathrm{O}$ trabalho colaborativo entre equipes de engenheiros e projetistas geograficamente distribuídos, tem o potencial de reduzir tempo para o desenvolvimento do produto, reduzindo custo e aumentando a qualidade do produto final. A formação de equipes de trabalho colaborativo proporciam processos de trabalhos mais eficientes, diminuem o número de viagens e aumentam de oportunidades de interação pessoal em diferentes campos de trabalho (JOHANSON \& TÖRLIND, 2004). Neste processo, importa a escolha da tecnologia a ser utilizada por estas equipes. O quadro 1 lista essas tecnologias.

Quadro 1 - Tecnologias para comunicação a distância.

\begin{tabular}{ll} 
Tecnologia & Forma de interação e comunicação \\
Telefone e E-mail & $\begin{array}{l}\text { Tecnologias amplamente utilizadas não só para trabalhos } \\
\text { colaborativos }\end{array}$ \\
\hline Videofone & $\begin{array}{l}\text { Utilização da infraestrutura de rede telefônica para } \\
\text { transmissão de imagens e voz }\end{array}$ \\
\hline Videoconferência & $\begin{array}{l}\text { Permite realizar reuniões a distância: conexões telefônicas } \\
\text { ISDN (Integrated Services Digital Network ou por IP (via } \\
\text { Internet) }\end{array}$ \\
\hline Redes Multicast & $\begin{array}{l}\text { Tecnologia para videoconferência resultante do esforço do } \\
\text { IETF (Internet Engineering Task Force) com uso mais } \\
\text { eficiente da rede }\end{array}$ \\
\hline DesktopVideoconferencing & $\begin{array}{l}\text { Permite realizar reuniões através do computador } \\
\text { pessoal/desktop e disponibiliza os arquivos, documentos, e- } \\
\text { mails, além de permitir navegar na internet. A comunicação } \\
\text { pode ser enriquecida através do uso de whiteboards, chats, } \\
\text { aplicações compartilhadas, etc }\end{array}$ \\
\hline Permite que ligações telefônicas possam ser feitas por \\
computador
\end{tabular}


empreendimento, tendo como principais funcionalidades: controle, armazenamento e visualização de documentos; comunicação (correio eletrônico, grupos de discussão, vídeoconferência, etc); automação dos processos e do fluxo de trabalho (workflow). (Nascimento, 2004)

\begin{tabular}{ll}
\hline Rede Wireless & $\begin{array}{l}\text { WaveLAN (redes wireless) soluções móveis para conexão à } \\
\text { internet. }\end{array}$
\end{tabular}

Fonte: baseado em Swedish University Computer Network (2006)

Whittaker et al., (1997) apud Johanson \& Törlind (2004) afirmam que a comunicação informal em equipes remotas pode ser apoiada por ferramentas de interação como telefones, e-mail, mensagens instantâneas, espaços de trabalho compartilhados e tecnologias de percepção (awareness). Dourish \& Belloti (1992); Ackerman \& Starr (1995) apud Johanson \& Törlind (2004) destacam que a percepção do progresso das atividades cria uma compreensão e conhecimento compartilhado das atividades dos outros membros, permitindo determinar o contexto das suas próprias atividades, objetivos e motivações.

A inovação nas atividades colaborativas ainda contam com uma arquitetura para colaboração móvel inteligente baseada em tecnologias Web Semântica, Web Services e Agentes (AZIZ et al., 2004). A Web Semântica é uma extensão da Web atual, onde a informação possui um significado bem definido, permitindo a cooperação entre computadores e pessoas. Portanto, segundo Hendler et al., (2002) apud Aziz et al., (2004) a Web Semântica é baseada na idéia de ter dados na web já definidos e relacionados de modo que possam ser usados mais eficientemente para: descoberta, automação, integração e reuso através de várias aplicações.

O Web Services é uma aplicação modular que possui um conteúdo e uma descrição própria, podendo ser publicada, locada e chamada através da web. Uma vez que um Web Service é desenvolvido, outras aplicações e outros Web Services podem descobrir e chamar os serviços, desconsiderando o sistema operacional e a linguagem de programação (Kreger, 2001 apud Aziz et al., 2004).

No ambiente móvel de construção, agentes (software) são essenciais para auxiliar as pessoas em relação aos dados e serviços específicos. Agente é um programa com conteúdo próprio capaz de controlar suas próprias tomadas de decisões e agir de acordo com sua percepção do seu ambiente, em busca de um ou mais objetivos. Os agentes garantem uma relação semântica aos dados de recursos, aumentando o nível de identificação do conhecimento e prevenindo o usuário de ser esmagado por informações irrelevantes através de filtros baseados em regras e adaptação da informação ao contexto e preferência individuais (AZIZ et al., 2004). Segundo 
Aziz et al. (2004), quando vários agentes são chamados para trabalharem juntos resulta em um sistema chamado "multi-agentes".

Portanto, tecnologias como Web Semântica, Web Services e Agentes possibilitam alavancar a capacidade da computação móvel na construção. Essas tecnologias têm o potencial de causar uma mudança de paradigma no gerenciamento da construção, permitindo o acesso do usuário móvel a uma grande variedade de dados e serviços quando necessário. Entretanto, a concretização dos serviços baseado na Web Semântica para computação móvel na construção devem satisfazer restrições introduzidas pela complexidade da tecnologia, custo, necessidades do usuário e interoperabilidade (AZIZ et al., 2004).

\section{O IMPACTO DAS NOVAS TECNOLOGIAS NA ESTRUTURA ORGANIZACIONAL}

Todas estas inovações requerem mudanças na estrutura organizacional dos setores que compõem a Indústria da Construção Civil. O advento dessas tecnologias surtiu impactos nos processos de desenvolvimento de projeto e na formação profissional.

Em relação aos processos de desenvolvimento de projetos a tecnologia alavancou processos da engenharia simultânea. Onde a engenharia simultânea (ES) é o processo no qual, grupos interdepartamentais trabalham de forma interativa e formal no projeto do ciclo de vida completo do produto ou serviço a fim de buscar e realizar a melhor combinação entre as metas de qualidade, custo e prazo (MUNIZ JR., 1995 apud FABRÍCIO, 2002).

A ES possibilitar redução de tempo de desenvolvimento de novos projetos, visando, antes da concorrência, o lançamento de novas tecnologias, produtos e serviços. Desta maneira procuram-se uma ampliação da competitividade, em virtude de maior agilidade na geração de projetos e uma flexibilidade produtiva, atendendo de modo ágil, as novas demandas de mercado (FABRÍCIO, 2002). Acrescenta o autor que incorpora um caráter multidisciplinar que considera os requisitos para a qualidade do produto e do processo de uma inovação. Desta maneira, o estudo multidisciplinar das inovações construtivas torna o processo da inovação mais ágil, assegurando maior confiabilidade, eficiência e eficácia para as ações de racionalização de propostas.

A aplicação dos conceitos de engenharia simultânea promove a entrada de inovações construtivas e requer mudanças na formação profissional, tanto para o exercício das atividades quanto para as técnicas e equipamentos utilizados. 
As mudanças que ocorrem na Construção Civil demandam o desenvolvimento de novas habilidades, conhecimentos, competências e comportamentos. Para tanto é necessário treinar e reconverter os profissionais do setor de modo que se adaptem ao contexto tecnológico em vigor (SILVA \& FREITAS, 1999).

No processo tradicional, o projeto é resultado de uma série de imagens estáticas como vistas, cortes, detalhes e perspectivas a fim de representar um único objeto. Esse processo é caracterizado pela sua linearidade, em que o principal obstáculo é a compreensão do conjunto da obra a ser construída. No novo ambiente de trabalho, em que se utiliza o computador, muda a forma de se pensar o espaço. Agora, é possível ter infinitas vistas de um mesmo objeto com os mais variados planos de projeção, tornando-se um processo não linear, em que é possível ter um controle e uma percepção global. Dessa maneira, um desenho pode ser utilizado várias vezes, acrescentando novos detalhes, novas informações e sendo impresso em outras escalas. O computador separa o sujeito do objeto, uma vez que o sujeito está no mundo físico e o objeto está no mundo virtual. Assim, o computador apartou três elementos que sempre estiveram juntos na representação arquitetônica: o olho, a mão e a base material de representação (CARVALHO \& ALMEIDA, 2002).

Por outro lado, trouxe beneficios pela possibilidade de ter um banco de dados que armazenasse as informações do projeto e permite a gestão do conhecimento de tudo que foi criado para o mesmo.

Quintão (2003) registra que a cada projeto surge um conhecimento para a organização que fica armazenado num banco de dados. Para cada projeto encerrado reune lições aprendidas pela avaliação do projeto sob a ótica do cliente e do produto.

Essa é um caminho novo, pela qual as empresas do setor terão que adaptar-se, pois ainda é objeto de estudos. Terra (2001) apud Yamauchi (2003) afirma que a gestão do conhecimento está relacionada com a habilidade das empresas em fazer uso e ligar diversas fontes e tipos de informação da organização, de modo a desenvolver competências específicas e aptidão inovadora consolidados em novos produtos, processos, sistemas de gerenciamento e liderança de mercado. Além de permitir a aplicação do conhecimento adquirido em novos projetos, a gestão do conhecimento também está relacionada com a geração de inovação em projetos.

Afim de complentar a discussão, quem atua no setor deverá ainda apresentar em seus projetos sua preocupação com uso de materiais e produtos sustentaveis. Surge a necessidade de buscar 
uma construção sustentável, desde a fase da concepção do projeto. Cabe destacar, neste artigo, pelo menos o conceito de desenvolvimento sustentável que se desenvolveu na década de 90 a partir da Conferência das Nações Unidas sobre o Meio Ambiente e Desenvolvimento, a Rio 92. Em 1987 divulgou-se o relatório da Comissão Mundial sobre o Meio Ambiente e Desenvolvimento - ou Comissão Brundtland, que define o desenvolvimento sustentável como "aquele que satisfaz as necessidades do presente sem comprometer a capacidade das gerações futuras de satisfazer suas próprias necessidades" (DIAS, 2002; EDWARDS \& HYETT, 2004, p.7).

\section{PROPOSTA DE UM CHECK LIST PARA AVALIAÇÃO DE INOVAÇÕES TECNOLÓGICA EM PROJETO}

A proposta do check list de inovações tecnológicas em projetos é uma listagem de mudanças relacionadas ao gerenciamento dos canteiros de obra. De acordo com os autores, a aplicação dessa listagem possibilita a análise sobre a implantação e gestão das inovações tecnológicas por empresas construtoras, resultando no desenvolvimento de novas ações estratégicas e mudanças futuras na organização.

Este trabalho quer possibilitar aos gestores de projetos a análise do nível de atualização em que se encontra a sua unidade e os produtos que entrega ao mercado. Para tanto, o documento de avaliação foi dividido em 4 partes: requisitos para o projeto (produto), infra-estrutura organizacional, ferramentas tecnológicas disponíveis e aplicação dos conceitos Desenvolvimento Sustentável nos projetos, conforme a Tabela 1.

A listagem deverá ser aplicada aos profissionais que atuam na área de projetos. No item "A" requisitos do projeto - o profissional avalia se as novas exigências de mercado, são contempladas e oferecem a possibilidade de personalizar ambientes internos e incorporar estratégias de flexibilidade para as construções.

No item "B" o projetista avaliará se os seus procedimentos organizacionais e métodos de trabalho estão de acordo com as melhores práticas do mercado. Assim, irá verificar se no seu escritório existem procedimentos para gerenciar a informação e o conhecimento, garantindo os menores custos, cumprimento de prazos e melhor desempenho na execução. Em relação aos métodos de trabalho, o profissional verificará se está incorporando processos de engenharia simultânea.

No item "C" são indicadas as ferramentas tecnológicas que possibilitam o desenvolvimento do processo de projeto de modo integrado, podendo analisar os vários aspectos do ciclo de 
vida da edificação. Neste item também são expostas ferramentas para o trabalho colaborativo e distribuído de equipes de projeto. Assim, o profissional é capaz de avaliar a sua posição em relação aos recursos tecnológicos disponíveis e caso esteja abaixo do desejado, é possível buscar soluções competitivas de mercado.

No item " $D$ " são observados conceitos para o desenvolvimento de um projeto sustentável. Avalia-se incorporação dos conceitos aos projetos, por exemplo, inserção de um sistema de baixo consumo energético e de manutenção da edificação ao longo do seu ciclo de vida, visando oferecer um projeto mais racional e com menor interferência ambiental.

Todos esses aspectos apresentados possibilitam os profissionais a observarem sua situação em relação as melhores práticas de mercado. Ainda, a incorporação dessas inovações no processo de projeto contribui para que o profissional participe do mercado de forma mais competitiva, com agregação de valor e qualidade ao seu produto.

Tabela 1 - Listagem para avaliação de inovações em projeto de edificações Baseado em: POZZOBON, FREITAS \& HEINECK (1999)

\begin{tabular}{|c|c|c|c|c|c|c|}
\hline \multirow{2}{*}{\multicolumn{7}{|c|}{ Inovações tecnológicas em projetos de edificações }} \\
\hline \multirow{2}{*}{\multicolumn{7}{|c|}{ 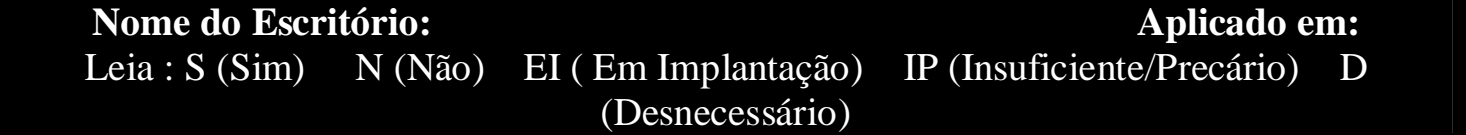 }} \\
\hline & & & & & & \\
\hline \multicolumn{7}{|l|}{ A - Requisito do projeto (produto) } \\
\hline 1. Para cada projeto é elaborado um programa de necessidades & $\mathrm{S}$ & $\mathrm{N}$ & $\mathrm{E}$ & $\mathrm{P}$ & & D \\
\hline $\begin{array}{l}\text { 2. Há documento para acompanhamento e atendimento ao programa de } \\
\text { necessidades }\end{array}$ & $\mathrm{S}$ & $\mathrm{N}$ & $\mathrm{E}$ & $\mathrm{P}$ & & D \\
\hline 3. Existe Projeto estrutural & $\mathrm{S}$ & $\mathrm{N}$ & $\mathrm{E}$ & $\mathrm{P}$ & & $\mathrm{D}$ \\
\hline 4. Existe Projeto de incêndio & $\mathrm{S}$ & $\mathrm{N}$ & E & $\mathrm{P}$ & & D \\
\hline 5. Existe Projeto de segurança & $\mathrm{S}$ & $\mathrm{N}$ & $\mathrm{E}$ & $\mathrm{P}$ & & D \\
\hline 6. Existem procedimentos e considerações sobre estanqueidade & $\mathrm{S}$ & $\mathrm{N}$ & $\mathrm{E}$ & $\mathrm{P}$ & & $\mathrm{D}$ \\
\hline 7. O projeto permite flexibilidade inicial? & $\mathrm{S}$ & $\mathrm{N}$ & $\mathrm{E}$ & $\mathrm{P}$ & & D \\
\hline 8. O projeto permite flexibilidade permanente? & $\mathrm{S}$ & $\mathrm{N}$ & $\mathrm{E}$ & $\mathrm{P}$ & & $\mathrm{D}$ \\
\hline 9. Utiliza soluções construtivas que permitem maior variação espacial & $\mathrm{S}$ & $\mathrm{N}$ & $\mathrm{E}$ & $\mathrm{P}$ & & $\mathrm{D}$ \\
\hline \multicolumn{7}{|l|}{ B - Procedimentos Organizacionais e Métodos de Trabalho } \\
\hline 1. Existem procedimentos para padronização dos projetos? & $\mathrm{S}$ & $\mathrm{N}$ & $\mathrm{E}$ & $\overline{\mathrm{P}}$ & & $\mathrm{D}$ \\
\hline 2. Existem procedimentos para compatibilização dos projetos? & $\mathrm{S}$ & $\mathrm{N}$ & $\mathrm{E}$ & $\mathrm{P}$ & & $\mathrm{D}$ \\
\hline
\end{tabular}




\begin{tabular}{|c|c|c|c|c|c|c|c|c|c|}
\hline \multirow{2}{*}{\multicolumn{9}{|c|}{\begin{tabular}{|c|c|} 
& \\
\end{tabular}}} & \\
\hline & & & & & & & & & \\
\hline & $\mathrm{S}$ & $\mathrm{N}$ & $\mathrm{E}$ & $\mathrm{P}$ & $\mathrm{D}$ & & & & D \\
\hline 4. As informações na apresentação dos projetos são claras? & $\mathrm{S}$ & $\mathrm{N}$ & $\mathrm{E}$ & $\mathrm{P}$ & & & & & D \\
\hline 5. Existem procedimentos para o detalhamento adequado do projeto? & $\mathrm{S}$ & $\mathrm{N}$ & E & $\mathrm{P}$ & & & & & $\mathrm{D}$ \\
\hline 6. Existem procedimentos para as Informações complementares? & $\mathrm{S}$ & $\mathrm{N}$ & $\mathrm{E}$ & $\mathrm{P}$ & & N & & & $\mathrm{D}$ \\
\hline 7. Existem procedimentos que facilitam a consulta de dados do projeto? & $\mathrm{S}$ & & $\mathrm{E}$ & $\mathrm{P}$ & $\mathrm{D}$ & $\mathrm{N}$ & & & $\mathrm{D}$ \\
\hline 8. Existem procedimentos para dar agilidade e cumprimento dos prazos? & $\mathrm{S}$ & & $\mathrm{E}$ & $\mathrm{P}$ & $\mathrm{D}$ & $\mathrm{N}$ & & & $\mathrm{D}$ \\
\hline 9. Existem procedimentos para elaboração de custos adequados ao projeto? & $\mathrm{S}$ & & $\mathrm{E}$ & $\mathrm{P}$ & $\mathrm{D}$ & $\mathrm{N}$ & & & $\mathrm{D}$ \\
\hline 10. Existem procedimentos para o acompanhamento da execução? & $\mathrm{S}$ & & $\mathrm{E}$ & $\mathrm{P}$ & $\mathrm{D}$ & $\mathrm{N}$ & & & $\mathrm{D}$ \\
\hline 11. Serviços de pós-venda dos projetistas? & $\mathrm{S}$ & $\mathrm{N}$ & $\mathrm{E}$ & $\mathrm{P}$ & & & & & $\mathrm{D}$ \\
\hline 12. São utilizadas as lições aprendidas em novos projetos? & $\mathrm{S}$ & $\mathrm{N}$ & $\mathrm{E}$ & $\mathrm{P}$ & $\mathrm{D}$ & $\mathrm{N}$ & & & $\mathrm{D}$ \\
\hline 13. No projeto são analisados todos os elementos da construção? & $\mathrm{S}$ & $\mathrm{N}$ & $\mathrm{E}$ & $\mathrm{P}$ & $\mathrm{D}$ & & & & $\mathrm{D}$ \\
\hline 14. No projeto são incluídos todos os departamentos organizacionais? & $\mathrm{S}$ & & $\mathrm{E}$ & $\mathrm{P}$ & $\mathrm{D}$ & $\mathrm{N}$ & & & $\mathrm{D}$ \\
\hline 15. No projeto são considerados aspectos de marketing? & $\mathrm{S}$ & & $\mathrm{E}$ & $\mathrm{P}$ & & $\mathrm{N}$ & & & $\mathrm{D}$ \\
\hline 16. No projeto são consideradas as solicitações dos clientes? & $\mathrm{S}$ & & $\mathrm{E}$ & $\mathrm{P}$ & & $\bar{N}$ & & & $\mathrm{D}$ \\
\hline 17. É feito Cronograma de atividade & $\mathrm{S}$ & & $\mathrm{E}$ & $\mathrm{P}$ & & $\mathrm{N}$ & & & $\mathrm{D}$ \\
\hline 18. Documentação técnica disponível em local visivel & $\mathrm{S}$ & & $\mathrm{E}$ & $\mathrm{P}$ & & $\mathrm{N}$ & & & $\mathrm{D}$ \\
\hline $\begin{array}{l}\text { 19. Há documentação para controle de qualidade de execução e } \\
\text { recebimento de serviços }\end{array}$ & $\mathrm{S}$ & $\mathrm{N}$ & $\mathrm{E}$ & $\mathrm{P}$ & & $\bar{N}$ & & & $\mathrm{D}$ \\
\hline C-Ferramentas Tecnológicas & & & & & & & & & \\
\hline 1. É utilizado algum sistema CAD2D? & $\mathrm{S}$ & $\mathrm{N}$ & $\mathrm{E}$ & $\mathrm{P}$ & & $\mathrm{N}$ & & & D \\
\hline 2. É utilizado algum sistema CAD3D? & $\mathrm{S}$ & & E & $\mathrm{P}$ & & $\mathrm{N}$ & & & $\mathrm{D}$ \\
\hline 3. É utilizado algum sistema CAD4D? & $\mathrm{S}$ & $\mathrm{N}$ & $\mathrm{E}$ & $\mathrm{P}$ & & $\mathrm{N}$ & & & $\mathrm{D}$ \\
\hline 4. É utilizado algum software paramétrico? & $\mathrm{S}$ & $\mathrm{N}$ & $\mathrm{E}$ & $\mathrm{P}$ & $\mathrm{D}$ & $\mathrm{N}$ & & & $\mathrm{D}$ \\
\hline 5. É utilizado telefone ou e-mail para comunicação? & $\mathrm{S}$ & $\mathrm{N}$ & $\mathrm{E}$ & $\mathrm{P}$ & $\mathrm{D}$ & $\mathrm{N}$ & & $\mathrm{P}$ & $\mathrm{D}$ \\
\hline 6. É utilizada infra-estrutura de videofone para comunicação? & $\mathrm{S}$ & $\mathrm{N}$ & $\mathrm{E}$ & $\mathrm{P}$ & D & & & & D \\
\hline
\end{tabular}




\begin{tabular}{|c|c|c|c|c|c|}
\hline 7. É utilizada sala de videoconfência para reuniões virtuais? & $\mathrm{S}$ & $\mathrm{N}$ & $\mathrm{E}$ & $\mathrm{P}$ & $\mathrm{D}$ \\
\hline 8. São utilizadas redes multicast para videoconferência? & $\mathrm{S}$ & $\mathrm{N}$ & $\mathrm{E}$ & $\mathrm{P}$ & $\mathrm{D}$ \\
\hline 9. É utilizada alguma aplicação para videoconferência no desktop? & $\mathrm{S}$ & $\mathrm{N}$ & $\mathrm{E}$ & $\mathrm{P}$ & $\mathrm{D}$ \\
\hline 10. É utilizada telefonia IP para comunicação? & $\mathrm{S}$ & $\mathrm{N}$ & $\mathrm{E}$ & $\mathrm{P}$ & $\mathrm{L}$ \\
\hline $\begin{array}{l}\text { 11. É utilizada alguma ferramenta do tipo Messaging System - Skype, } \\
\text { MSN, ICQ? }\end{array}$ & $\mathrm{S}$ & $\mathrm{N}$ & $\mathrm{E}$ & $\mathrm{P}$ & $\mathrm{D}$ \\
\hline 12. É utilizada extranet na gerencia de projeto? & $\mathrm{S}$ & $\mathrm{N}$ & $\mathrm{E}$ & $\mathrm{P}$ & $\mathrm{D}$ \\
\hline 13. É utilizada rede wireless? & $\mathrm{S}$ & $\mathrm{N}$ & $\mathrm{E}$ & $\mathrm{P}$ & $\mathrm{D}$ \\
\hline 14. É utilizado protocolo SIP para reuniões virtuais? & $\mathrm{S}$ & $\mathrm{N}$ & $\mathrm{E}$ & $\mathrm{P}$ & $\mathrm{D}$ \\
\hline 15. É utilizada Web Semântica para sistemas de colaboração? & $\mathrm{S}$ & $\mathrm{N}$ & $\mathrm{E}$ & $\mathrm{P}$ & $\mathrm{D}$ \\
\hline 16. É utilizado Web Services para sistemas de colaboração? & $\mathrm{S}$ & $\mathrm{N}$ & $\mathrm{E}$ & $\mathrm{P}$ & $\mathrm{D}$ \\
\hline 17. É utilizada tecnologia de Agentes para sistemas de colaboração? & $\mathrm{S}$ & $\mathrm{N}$ & $\mathrm{E}$ & $\mathrm{P}$ & D \\
\hline 18. É utilizado banco de dados? & $\mathrm{S}$ & $\mathrm{N}$ & $\mathrm{E}$ & $\mathrm{P}$ & $\mathrm{D}$ \\
\hline 19. É utilizado Sistemas de Gestão do Conhecimento? & $\mathrm{S}$ & $\mathrm{N}$ & $\mathrm{E}$ & $\mathrm{P}$ & $\mathrm{D}$ \\
\hline \multicolumn{6}{|c|}{ D - Aplicação dos conceitos de Desenvolvimento Sustentável nos projetos } \\
\hline 1. É considerado o ciclo de vida de materiais na sua seleção para o projeto & $\mathrm{S}$ & $\mathrm{N}$ & $\mathrm{E}$ & $\mathrm{P}$ & D \\
\hline $\begin{array}{l}\text { 2. Os processos construtivos são escolhidos porque gera pouco consumo } \\
\text { de energia }\end{array}$ & $\mathrm{S}$ & $\mathrm{N}$ & $\mathrm{E}$ & $\mathrm{P}$ & $\mathrm{D}$ \\
\hline 3. Há preocupação com as perdas nos processos construtivos & $\mathrm{S}$ & $\mathrm{N}$ & $\mathrm{E}$ & $\mathrm{P}$ & $\mathrm{D}$ \\
\hline 4. Há preocupação nos impactos com o meio ambiente & $\mathrm{S}$ & $\mathrm{N}$ & $\mathrm{E}$ & $\mathrm{P}$ & $\mathrm{D}$ \\
\hline 5. Há opções de escolha dos materiais de fácil manutenção & $\mathrm{S}$ & $\mathrm{N}$ & $\mathrm{E}$ & $\mathrm{P}$ & $\mathrm{D}$ \\
\hline 6. Uso de sistemas eficientes de iluminação, ventilação e ar condicionado. & $\mathrm{S}$ & $\mathrm{N}$ & $\mathrm{E}$ & $\mathrm{P}$ & $\mathrm{D}$ \\
\hline 7. Há preocupação com o aproveitamento da água da chuva & $\mathrm{S}$ & $\mathrm{N}$ & $\mathrm{E}$ & $\mathrm{P}$ & $\mathrm{D}$ \\
\hline 8. Considerações sobre a possibilidade de expansão e modernização & $\mathrm{S}$ & $\mathrm{N}$ & $\mathrm{E}$ & $\mathrm{P}$ & $\mathrm{D}$ \\
\hline $\begin{array}{l}\text { 9. Facilidade de acesso às instalações hidráulicas, elétricas, } \\
\text { condicionamento de ar e aquecimento de água. }\end{array}$ & $\mathrm{S}$ & $\mathrm{N}$ & $\mathrm{E}$ & $\mathrm{P}$ & $\mathrm{D}$ \\
\hline $\begin{array}{l}\text { 10. Especificação de materiais que sejam re-aproveitáveis no todo ou em } \\
\text { parte, duráveis, desmontáveis e recicláveis. }\end{array}$ & $\mathrm{S}$ & $\mathrm{N}$ & $\mathrm{E}$ & $\mathrm{P}$ & $\mathrm{D}$ \\
\hline 11. Utilização de ferramentas de auxílio na seleção de materiais & $\mathrm{S}$ & $\mathrm{N}$ & $\mathrm{E}$ & $\mathrm{P}$ & \\
\hline
\end{tabular}




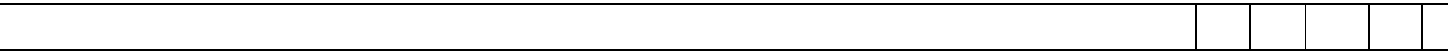

\section{CONSIDERAÇÕES FINAIS}

As inovações tecnológicas em projetos surgem de modo a atender às novas exigências do mercado, que abrangem qualidade, agilidade, atendimento às necessidades do cliente, flexibilidade e desenvolvimento sustentável das construções. Dentro desse contexto são apresentadas as ferramentas de tecnologia da informação, que auxiliam o atendimento dessas inovações. Estas convergem para um novo paradigma de projeto, em que na fase de concepção são considerados todos os elementos do ciclo de vida da edificação, incluindo os profissionais envolvidos, departamentos organizacionais, aspectos da produção, operação, manutenção e demolição. Nesse modelo holístico, são também analisados os aspectos da inserção da edificação no ambiente e seu impacto, contribuindo para o desenvolvimento sustentável das construções.

A listagem proposta neste trabalho sintetiza a pesquisa bibliográfica realizada e sua aplicação possibilita ao profissional do setor verificar a sua situação em relação aos novos procedimentos de trabalho. Desta maneira possibilita a tomada de decisões estratégicas e a adoção das inovações tecnológicas no setor de projetos.

As ações de arquitetos e engenheiros têm um papel relevante para aplicar os conceitos apresentados à produção do ambiente construído. Desta forma o profissional poderá implementar as inovações, atender de modo competitivo às demandas do mercado e trazer benefícios ao meio ambiente, ao homem e ao desenvolvimento econômico e tecnológico.

\section{REFERENCIAS}

AZIZ, Z; ANUMBA, C; RUIKAR, D; CARRILO, P; BOUCHLAGHEM, D. Semantic web basead services for intelligent móbile construction collaboration. Electronic Journal of Information Technology in Construction (Itcon), v. 9, p. 367-379, 2004.

BRANDÃO, D. Q. Diversidade e potencial de flexibilidade de arranjos espaciais de apartamentos: uma análise do produto imobiliário no Brasil. Florianópolis, 2002. Tese (Programa de Pós-Graduação em Engenharia de Produção) - Universidade Federal de Santa Catarina.

CARVALHO, F.C.A. Gestão do Conhecimento: $O$ caso de uma empresa de alta tecnologia. Florianópolis, 2000. Dissertação (Mestrado em Engenharia da Produção) UFSC.

CARVALHO, G. L.; ALMEIDA, I. A. C. A representação do projeto arquitetônico aliado a computação gráfica aos traçados de desenhos tradicionais. In: XIV Congreso Internacional de Ingeniería Gráfica. Santander, Espana, 2002. 
DEGANI, C. M.; CARDOSO, F. F. A sustentabilidade ao longo do ciclo de vida de edifícios: a importância da etapa de projeto arquitetônico. In: NUTAU'2002. S. Paulo, SP. 2002. p. 1347-1357.

EDWARDS, B.; HYETT, P. Guia Básica de la Sostenibilidad. Gustavo Gili, Barcelona, 2004.

FABRICIO, M. M. Projeto Simultâneo na Construção de Edifícios. São Paulo, 2002. Tese (Doutorado em Engenharia) - Departamento de Engenharia de Construção Civil, USP.

FU, C; AOUAD, G; LEE, A; MASHALL-PONTING, A; WU, S. IFC model viewer to support nD model application. Automation in Construction, v. 15, p. 178-185, 2006.

GRILO, L. M. ; PEÑA, M. D. ; SANTOS, L. A.; FILIPPI, G. ; MELHADO, S. B. Implementação da gestão da qualidade em empresas de projeto. Ambiente Construído, Porto Alegre, v.3, n.1. jan./mar. 2003.

JACOSKI, C. A. Integração e interoperabilidade em projetos de edificações - Uma Implementação com IFC/ XML. Florianópolis, 2003. Tese do Programa de Pós-Graduação em Engenharia de Produção e Sistemas da Universidade Federal de Santa Catarina.

JOHANSON, M; TÖRLIND, P. Mobility support for distributed collaborative teamwork. Electronic Journal of Information Technology in Construction (Itcon), v. 9, p. 355-366, 2004.

MARSHALL-PONTING, A. J; AOUAD, G. An nD modeling approach to improve communication processes for construction. Automation in Constructio, v. 14, p. 311-321, 2005.

MELHADO, S. B. Qualidade do projeto na construção de edifícios: aplicação ao caso das empresas de incorporação e construção. 1994. Tese (Doutorado) - Universidade de São Paulo, São Paulo.

MELHADO, S. B. O plano da qualidade dos empreendimentos e a engenharia simultânea na construção de edifícios. In: ENCONTRO NACIONAL DE ENGENHARIA DE PRODUÇÃO, 1999, Rio de Janeiro. Anais... Rio de Janeiro: UFRJ/ABEPRO, 1999.

NASCIMENTO, L. A. Proposta de um sistema de recuperação de informação para extranet de projeto. São Paulo, 2004. Dissertação (Mestrado em Engenharia) - Universidade de São Paulo.

PINHO, A. C.; FREITAS, U. C.; TRAMONTANO, M. A modelagem digital aplicada ao processo de projeto de edifícios de apartamentos. In: III WORKSHOP BRASILEIRO DE GESTÃO DO PROCESSO DE PROJETO NA CONSTRUÇÃO DE EDIFÍCIOS, 3., 2003, Belo Horizonte. Anais...

POZZOBON, C. E; FREITAS, M. C. D; HEINECK, L. F. Listagem de mudanças relacionadas ao gerenciamento dos canteiros de obras. In: I Simpósio Brasileiro de Gestão da Qualidade na Construção Civil, 1, 1999, Recife. 
QUINTÃO, F. B. M. A gestão de pessoas e a gestão do conhecimento adquirindo papel fundamental no processo de gestão de projetos. In: III Workshop Brasileiro de Gestão do Processo de Projeto na Construção de Edifícios, 3., 2003, Belo Horizonte. Anais...

SHANIAN, A.; SAVADOGO, O. A material selection model based on the concept of multiple attribute decision making. Materials and Design 27, 2006. 329-337.

SILVA, C. R. O; FREITAS, M. C. D. Reconversion profesional en el contexto de la construccion civil. In: Congresso Iberoamericano de Enseñanza de la Ingeniería y la Arquitectura - UNIAICC, Havana/Cuba , 1999.

SOUZA, A. F; COELHO, R. T. Tecnologia CAD/CAM - Definições e estado da arte visando auxiliar sua implantação em um ambiente fabril. In: XXIII ENEGEP, 2003, Ouro Preto.

SUZUKI, M.; OKA, T. Estimation of life cycle energy consumption and CO2 emission of office buildings in Japan. Energy and Buildings, 28, 1998. 3341

SWEDISH UNIVERSITY COMPUTER NETWORK. Historical Technology. Disponível em < http://www.meetings.sunet.se/history.php?lang=en> Acesso em: 08 nov. 2006.

TANYER, A. M; AOUAD, G. Moving beyond the fourth dimension with an IFC-based single project database. Automation in Construction, v. 14, p. 15-32, 2005.

TSE, T. K; WONG, K. A; WONG, K. F. The utilization of building information models in $n D$ modelling: a study of data interfacing and adoption barriers. Electronic Journal of Information Technology in Construction (Itcon), v. 10, p. 85-110, 2005.

VEERAMANI, D; TSERNG, H. P; RUSSELL, J. S. Computer-integrated collaborative design and operation the construction industry. Automation in Construction, v. 7, p. 485-492, 1998.

YAMAUCHI, V. Implementação de inovação em projetos através da gestão do conhecimento. In: Simpósio Brasileiro de Gestão e Economia da Construção, 3., 2003, São Carlos, SP. 